\title{
Double Dissociation of Basolateral and Central Amygdala Lesions on the General and Outcome-Specific Forms of Pavlovian-Instrumental Transfer
}

\author{
Laura H. Corbit and Bernard W. Balleine \\ Department of Psychology and the Brain Research Institute, University of California, Los Angeles, Los Angeles, California 90095
}

\begin{abstract}
This series of experiments compared the effects of lesions of the basolateral complex (BLA) and the central nucleus (CN) of the amygdala on a number of tests of instrumental learning and performance and particularly on the contribution of these structures to the specific and general forms of pavlovian-instrumental transfer (PIT). In experiment 1, groups of BLA-, CN-, and sham-lesioned rats were first trained to press two levers, each earning a unique food outcome (pellets or sucrose), after which they were given training in which two auditory stimuli (tone and white noise) were paired with these same outcomes. Tests of specific satiety induced outcome devaluation, and tests of PIT revealed that, although the rats in all of the groups performed similarly during both the instrumental and pavlovian acquisition phases, BLA, but not CN, lesions abolished selective sensitivity to a change in the reward value of the instrumental outcome as well as to the selective excitatory effects of reward-related cues in PIT. In experiment 2, we developed a procedure in which both the general motivational and the specific excitatory effects of pavlovian cues could be assessed in the same animal and found that BLA lesions abolished the outcome-specific but spared the general motivational effects of pavlovian cues. In contrast, lesions of $\mathrm{CN}$ abolished the general motivational but spared the specific effects of these cues. Together, these results suggest that the BLA mediates outcome-specific incentive processes, whereas $\mathrm{CN}$ is involved in controlling the general motivational influence of reward-related events.
\end{abstract}

Key words: goal-directed action; instrumental conditioning; lever pressing; outcome devaluation; reward; pavlovian conditioning; transfer of control; general arousal; rat

\section{Introduction}

In recent years, it has become clear that, in instrumental conditioning, rats encode the relationship between their actions and the consequences or outcome of those actions (for review, see Dickinson and Balleine, 1994, 1995, 2002; Balleine, 2001, 2004). Furthermore, recent evidence suggests that choice between actions also depends on two forms of incentive learning: instrumental incentive learning, through which animals encode the value of the outcomes of their actions, and pavlovian incentive learning, which mediates the excitatory effects of stimuli (S) associated with sensory and general motivational aspects of rewarding events (for review, see Balleine, 2001, 2004; Dickinson and Balleine, 2002).

Advances have also been made in our understanding of the neural bases of incentive learning processes (Dayan and Balleine, 2002). For example, recent studies have established that the influence of reward and signals that predict reward depends on the amygdala. Nevertheless, there has been some disagreement on the locus of this influence within the amygdala itself. Balleine et

Received Nov. 2, 2004; revised Dec. 9, 2004; accepted Dec. 10, 2004.

This work was supported by a grant from the National Institute of Mental Health (NIMH 56446) to B.W.B.

Correspondence should be addressed to Bernard W. Balleine, Department of Psychology, University of California,

Los Angeles, Box 951563, Los Angeles, CA 90095. E-mail: balleine@psych.ucla.edu.

DOI:10.1523/JNEUROSCI.4507-04.2005

Copyright $\odot 2005$ Society for Neuroscience $\quad$ 0270-6474/05/250962-09\$15.00/0 al. (2003) reported evidence that lesions of the basolateral amygdala (BLA) abolish the sensitivity of instrumental performance to changes in the value of the instrumental outcome using a selective devaluation protocol. Furthermore, using a pavlovianinstrumental transfer (PIT) design, Blundell et al. (2001) found that the sensory-specific excitatory effects of reward-related cues on actions that earn the same outcome predicted by a stimulus was also abolished by lesions of the BLA. In contrast, both Hall et al. (2001) and Holland and Gallagher (2003) reported that lesions of the BLA had no effect on PIT but, rather, that it was lesions of the amygdala central nucleus $(\mathrm{CN})$ that were effective in eliminating this effect.

One critical difference between these reports lies in the training conditions, and potentially the forms of PIT, that were generated in these experiments. Both Balleine et al. (2003) and Blundell et al. (2001) trained their rats on two actions rewarded by different outcomes and assessed the effects of their devaluation and transfer manipulations on the choice between actions mediated by the sensory features of the outcomes associated with the actions and conditioned stimuli (CSs). In contrast, both Hall et al. (2001) and Holland and Gallagher (2003) trained their rats on a single lever and assessed the influence of a single excitatory cue on performance on that lever, a procedure that is likely to generate transfer based on the general motivational or affective significance of the pavlovian excitor (Dickinson and Dawson, 1987; Balleine, 1994). 
Experiment 1 compared the effects of BLA and CN lesions on both selective outcome devaluation and on the selective form of PIT. In experiment 2, we modified the selective PIT protocol to add a cue that predicted an outcome not earned by either instrumental action to generate, within subjects, both selective and general PIT. If it is the form of PIT that is responsible for the differences in reported results, lesions of the BLA should abolish outcome-specific PIT but spare general PIT. Conversely, lesions of CN should be expected to a spare outcome-specific PIT but abolish general PIT.

\section{Materials and Methods}

Subjects and apparatus. A total of 84 experimentally naive male LongEvans rats served as subjects; 48 animals were used in experiment 1, and 36 animals were used in experiment 2 . The rats were housed singly and were handled daily for 1 week before surgery. Training and testing took place in 24 Med Associates (East Fairfield, VT) operant chambers housed within sound- and light-resistant shells. Each chamber was equipped with a pump, which was fitted with a syringe that delivered $0.1 \mathrm{ml}$ of a $20 \%$ sucrose solution into a recessed magazine in the chamber. Each chamber was also equipped with a pellet dispenser that delivered one 45 mg pellet (Bio-Serve, Frenchtown, NJ) when activated. The chambers contained two retractable levers that could be inserted to the left and right of the magazine. The boxes also contained a white-noise generator, a sonalert that delivered a $3 \mathrm{kHz}$ tone, and a solanoid that, when activated, delivered a $5 \mathrm{~Hz}$ clicker stimulus. All stimuli were adjusted to $80 \mathrm{~dB}$ in the presence of background noise of $60 \mathrm{~dB}$ provided by a ventilation fan. A $3 \mathrm{~W}, 24 \mathrm{~V}$ houselight mounted on the top-center of the wall opposite the magazine provided illumination. Microcomputers equipped with the MED-PC program (Med Associates) controlled the equipment and recorded the lever presses.

Surgery. At the time of surgery, the average rat weighed $540 \mathrm{~g}$. The subjects received cell-body lesions of the BLA, the CN, or sham surgery. Rats were anesthetized using sodium pentobarbital (Nembutal; $50 \mathrm{mg} /$ $\mathrm{kg}$; Abbott Laboratories, Chicago, IL) and treated with atropine (0.1 mg) and were then placed in a stereotaxic frame (Stoelting, Wood Dale, IL) with the incisor bar set at $-3.3 \mathrm{~mm}$. The scalp was retracted to expose the skull, and small burr holes were drilled above the target regions. For lesions of the BLA, animals received bilateral injections of $0.25 \mu \mathrm{l}$ of 0.12 M NMDA in four sites (two per side) using a $1 \mu$ l Hamilton syringe [coordinates relative to bregma except ventral relative to dura; anteroposterior (AP), $-2.3,-3.0$; mediolateral $(\mathrm{ML}), \pm 5.2$; dorsoventral (DV), -7.6]. Each injection was made over $2 \mathrm{~min}$ and allowed to diffuse for an additional $2 \mathrm{~min}$ before removal of the needle. For lesions of the $\mathrm{CN}$ region, animals received $0.2 \mu \mathrm{l}$ injections of $0.01 \mathrm{M}$ AMPA at two sites (one per side: AP, -2.3 ; ML, $\pm 4.0 ; \mathrm{DV},-7.6$ ). Injections were again made over 2 min with an additional 2 min allowed before any movement of the needle. Animals in the surgical control group underwent similar treatment, except that no neurotoxin was injected; one-half had the needle lowered into each of the surgical placements of the other groups.

Histology. At the end of the experiment, the animals were killed using a lethal barbiturate overdose and were perfused transcardially with $0.9 \%$ saline followed by $10 \%$ formaldehyde solution. The brains were stored in $10 \%$ formalin solution for at least $48 \mathrm{~h}$ and then transferred to a $25 \%$ sucrose-formalin solution before $40 \mu \mathrm{m}$ coronal sections were cut throughout the region of the amygdala. Alternate slices were stained using thionin. Slides were examined for placement and extent of the lesion, with the latter assessed by microscopically examining sections for areas of marked cell loss as well as general shrinkage of a region relative to sham controls.

\section{Experiment 1: general behavioral procedures}

Training. After recovery from surgery, subjects were placed on a food deprivation schedule such that they received $15 \mathrm{~g}$ of their maintenance diet daily to maintain them at $\sim 85 \%$ of their free-feeding weight. The animals were fed after the training sessions of the day. Animals had ad libitum access to tap water while in the home cage. Each session started with the illumination of the houselight and insertion of the levers where appropriate and ended with the retraction of the levers and turning off of the houselight. All sessions were $30 \mathrm{~min}$ in duration unless otherwise stated.

Magazine training. Initially, all subjects received two sessions of magazine training in which the pellet and sucrose outcomes were delivered on independent random time (RT) $60 \mathrm{~s}$ schedules with the levers withdrawn.

Lever training. The animals were next trained on random ratio (RR) schedules of reinforcement. Each lever was trained separately and for one-half of the animals in each group; the left lever earned pellets, and the right lever earned the sucrose solution. The remaining animals received the opposite action-outcome pairings. The animals first received $1 \mathrm{~d}$ of continuous reinforcement and were then shifted to a RR -5 schedule (i.e., each action delivered an outcome with a probability of 0.2 ). After $4 \mathrm{~d}$ of training, this was changed to a RR-10 (or a probability of 0.1) schedule for an additional $4 \mathrm{~d}$. The animals received two training sessions each day, one with each action-outcome pair. The animals had a break of at least $1 \mathrm{~h}$ between sessions, and the order of sessions was alternated each day.

\section{Experiment 1: outcome devaluation}

Devaluation extinction tests. On the day after the final training session, all of the rats were given ad libitum access to one of the two outcomes for $1 \mathrm{~h}$. One-half of the animals in each action-outcome pair assignment received pellets ( $50 \mathrm{~g}$ placed in a bowl in the home cage), and the remaining animals received sucrose $(50 \mathrm{ml}$ in a drinking bottle fixed to the front of the home cage). Immediately after the prefeeding, the animals were placed in the operant chambers. A 10 min choice extinction test was then conducted, in which both levers were extended and the number of presses was counted on each lever. No outcomes were delivered during the test. After the first devaluation test, the animals received $1 \mathrm{~d}$ of retraining (RR-10; one session for each action-outcome pair) and were then given a second devaluation test on the following day. The second test was identical to the first, except that those animals that had had pellets devalued in the first test now had sucrose devalued, and those that had had sucrose devalued previously now had pellets devalued.

Devaluation rewarded test. After the second devaluation test conducted in extinction, the animals were retrained (RR-10; one $30 \mathrm{~min}$ session for each action-outcome pair) and on the following day were tested on both actions concurrently for their sensitivity to the devaluation manipulation when performance was rewarded. This test was conducted in the same manner as the extinction test, except that the outcomes were delivered as a consequence of instrumental performance. In this $10 \mathrm{~min}$ session, the two outcomes were delivered on independent ratio schedules (RR-10).

Consumption test of specific satiety. To assess the ability of the rats to distinguish between the two outcomes, they were given a consumption version of the specific-satiety devaluation test. One of the two outcomes was devalued in the same manner as in the tests described above, but now the dependent measure used was consumption of either that same outcome or a different outcome. Normal performance on this test (i.e., decreased consumption of the same food item relative to a different food) requires that the animals can distinguish between the two food items. The animals received two tests. In each test, they received $1 \mathrm{~h}$ of ad libitum access to one of the foods, either pellets or sucrose, in the home cage. The prefed food was removed, and then the animals were presented with the test food, either the sucrose or pellets, for an additional $20 \mathrm{~min}$, and their consumption in that test period was recorded. In the first test, one-half of the animals received the same food as that which they had just had access to and for the remaining animals, the different food was presented. In the second test, the animals were prefed the same food as in the first test; however, the food item presented in the test period was the opposite to that of the first test. As a result, animals that had received the same food during the prefeeding and testing in the first test now received the different food during the consumption test. Those that had previously received the different food during the first test now received the same food during the prefeeding and consumption test.

\section{Experiment 1: pavlovian-instrumental transfer-selective transfer test}

Pavlovian training. After the instrumental training and tests described above, the rats received eight sessions of pavlovian conditioning. Two 
auditory stimuli [tone (T) and white noise $(\mathrm{N})$ ] served as CSs and were paired with either pellet or sucrose delivery. For one-half of the rats in each lesion condition, the tone was paired with pellet delivery, and the noise was paired with sucrose delivery. The remaining half received the reverse pairings. Four presentations of each stimulus were given in each session in random order interspersed with periods in which no stimuli were present. The length of the intertrial intervals varied, but on average, these intervals were $5 \mathrm{~min}$. The stimuli presentations were 2 min long, during which the appropriate outcome was delivered on a RT-30 s schedule. The number of magazine entries during the stimuli as well as in a prestimulus interval of equal length ( $2 \mathrm{~min})$ was measured.

Transfer test. The animals received two extinction tests (one on each lever), $1 \mathrm{~d}$ apart. During each test, one of the levers was available, and each stimulus was presented four times interspersed with intervals of no stimulus $(\varnothing)$. Each test was $40 \mathrm{~min}$ in duration. In the first 8 $\mathrm{min}$, the levers were available, but no stimuli were presented. This period was followed by 16 , 2 min bins and contained a total of eight stimulus trials (four tone trials and four noise trials intermixed with eight $\varnothing$ trials in the following order: T, N, N, T, N, T, T, N).

\section{Experiment 2: pavlovian-instrumental}

transfer-general and selective transfer test

Instrumental training. In experiment 2 , the sur-

gical procedures were as described above; again, rats were given either sham, BLA, or CN lesions (36 rats total; 12 per group). After recovery from surgery, the rats received training in the same manner as outlined above, except that there were three possible earned outcomes: pellets, $20 \%$ sucrose, and $20 \%$ polycose plus $0.9 \%$ sodium chloride. Any given animal earned two of these outcomes, one by performance of the left lever response and one after performance of the right lever response. These assignments were counterbalanced within each lesion condition. Rats received $1 \mathrm{~d}$ of training on continuous reinforcement, $3 \mathrm{~d}$ of RR-5, and $3 \mathrm{~d}$ of RR-10 schedules of reinforcement.

Pavlovian training. After instrumental training, the animals received 10 sessions of pavlovian conditioning. The three auditory stimuli (tone, white noise, and clicker) served as CSs and were paired with either pellet, sucrose, or polycose delivery (counterbalanced). Four presentations of each stimulus were given in each session in random order interspersed with periods in which no stimuli were presented. The length of the intertrial intervals varied, but on average, these intervals were $5 \mathrm{~min}$. The stimuli presentations were 2 min long, during which the appropriate outcome was delivered on a RT-30 s schedule. The number of magazine entries during the stimuli as well as in a prestimulus interval of equal length (2 min) was measured.

Transfer test. The animals received two extinction tests (one on each lever), $1 \mathrm{~d}$ apart. During each test, one of the levers was available, and each of the three stimuli was presented three times interspersed with intervals of no stimulus $(\varnothing)$. Each test was $44 \mathrm{~min}$ in duration. In the first $8 \mathrm{~min}$, the levers were available, but no stimuli were presented. This period was followed by $18 \times 2 \mathrm{~min}$ bins that contained a total of nine stimulus trials (three trials for each stimulus) intermixed with $\varnothing$ trials. The order of the trials was randomly determined by the computer program.

\section{Results}

\section{Histology}

No recovery problem or weight loss was observed after surgery. Figure 1 displays the maximum and minimum damage resulting from the lesions for the rats included in the behavioral analyses for the CN (left) and BLA (right) based on the stereotaxic atlas of the rat brain by Paxinos and Watson (1998). Rats with unilateral damage or damage outside the target region were excluded from the behavioral analyses. In experiment 1, two animals were excluded from each the BLA and CN groups for such damage. Additionally, two animals from each the BLA and sham groups were excluded for biased responding in instrumental acquisition (i.e., these animals responded on one but not both levers in training). After these exclusions, the subject totals were as follows: CN, 10; BLA, 20; sham, 10. In experiment 2, two animals from each the $\mathrm{CN}$ and BLA groups were excluded for unilateral damage of the target nucleus resulting in the following group sizes: CN, 10; BLA, 10; sham, 12. BLA lesions typically created damage throughout the majority of the rostro-caudal extent of the BLA with some sparing of tissue in the caudal BLA and included the basal, accessory basal, and lateral divisions of the region. $\mathrm{CN}$ lesions generally included damage to the central, medial, and lateral divisions of this nucleus. No damage to the BLA was seen in this group. Figure 2 shows photomicrographs of representative lesions from each lesion condition.

\section{Experiment 1: effect of BLA and CN lesions on tests of instrumental conditioning}

\section{Instrumental training}

The number of lever presses per minute across days of training is displayed in the left panel of Figure 3. Rats in each of the groups acquired the instrumental responses, and their response rates increased across days. ANOVA conducted on the training data revealed no effect of group $\left(F_{(2,37)}=1.04 ; p>0.05\right)$ but a significant effect of training day $\left(F_{(8,296)}=64.11 ; p<0.01\right)$ and no interaction between group and day $\left(F_{(16,296)}=1.13 ; p>0.05\right)$, suggesting that all groups acquired instrumental conditioning at a similar rate. 


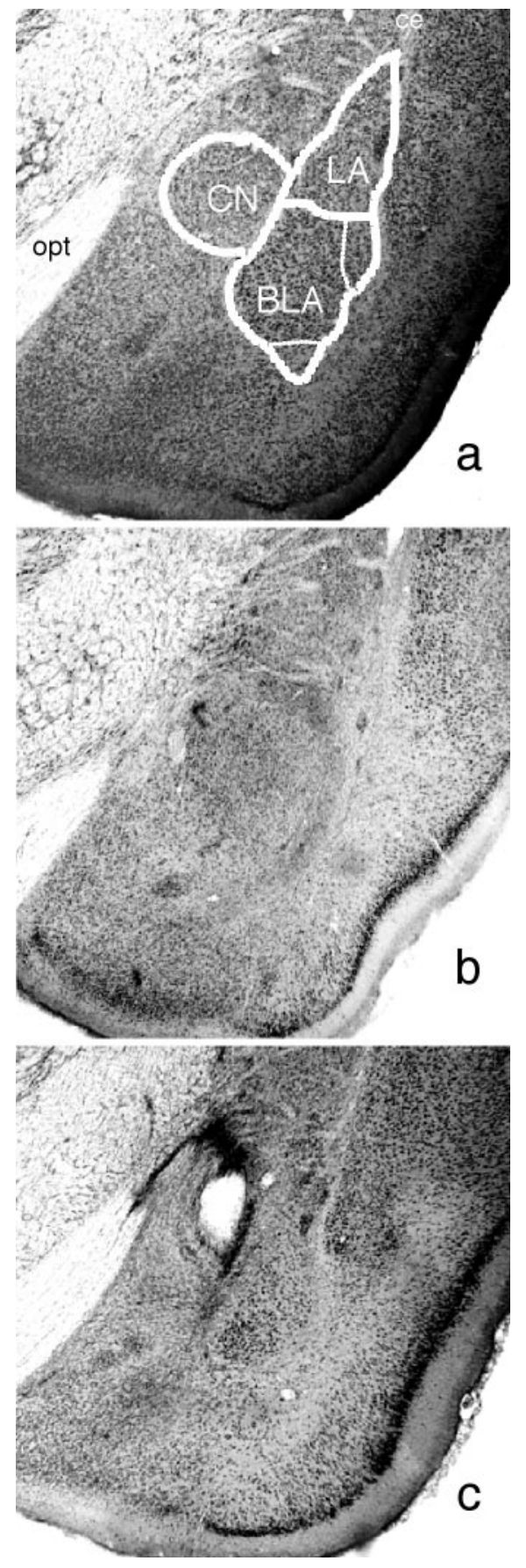

Figure 2. Low-power photomicrograph showing, in $a$, a sham brain on which the major boundaries of the basolateral complex [comprising the basolateral and lateral amygdala (LA)] and of the $\mathrm{CN}$ have been marked along with the optic tract (opt) and external capsule (ec) as general landmarks, in $b$, a representative cell-body lesion of the basolateral complex, and in c, a representative cell-body lesion of the central nucleus. These coronal sections are all approximately $-2.8 \mathrm{~mm}$ posterior to bregma.

\section{Devaluation extinction tests}

The mean number of lever presses per minute during the choice extinction test is presented in the right panel of Figure 3. As this figure makes clear, a devaluation effect was found in both the sham-lesioned rats and rats with CN lesions; these rats performed fewer of the action that in training had delivered the now devalued outcome than the other action. In contrast, the BLA-lesioned rats responded at similar rates on the two levers and failed to show a selective devaluation effect. This description was con- firmed by the statistical analysis, which revealed no effect of group $\left[F_{(2,37)}=1.46 ; p>0.05\right.$; mean squared error $(\mathrm{MSE})=$ 5630.1 but a significant effect of outcome (valued vs devalued) $\left(F_{(1,37)}=43.26 ; p<0.01\right)$ and an interaction between outcome and group $\left(F_{(2,37)}=6.0 ; p<0.01\right)$. Simple effects analysis revealed that, whereas a significant devaluation effect was present in the sham $\left(F_{(1,9)}=17.06 ; p<0.01\right)$ and $\mathrm{CN}$ rats $\left(F_{(1,9)}=8.15\right.$; $p<0.05)$, the response rates on the two levers did not differ in the BLA rats $\left(F_{(1,19)}=2.79 ; p>0.05\right)$.

\section{Rewarded test}

Performance in the rewarded test is shown in Figure 4. As in the extinction test, a robust devaluation effect was observed in the sham and $\mathrm{CN}$ rats; both groups performed fewer responses on the lever that delivered the devalued outcome. Again, even when the outcomes were delivered during the test, the BLA animals appeared to show less selective responding than the other groups, although, clearly, their performance improved when the outcomes were delivered relative to their performance in the extinction test. Additional analysis of the data established that this effect emerged across minutes of the session, suggesting that feedback from outcome delivery was an important factor in directing the performance of the BLA animals. The statistical analysis mostly confirmed this description. Whereas there was no main effect of group $\left(F_{(2,37)}=2.43 ; p>0.05\right.$; MSE $\left.=334515.4\right)$, there was a significant effect of outcome $\left(F_{(1,37)}=39.75 ; p<0.01\right)$. Although, in this test, the interaction between group and outcome was only marginally significant $\left(F_{(2,37)}=3.17 ; p=0.054\right)$, there was a significant effect of minute, suggesting that performance increased across minutes of the test $\left(F_{(9,333)}=6.56 ; p<0.01\right)$, and, although minute did not interact with group, there was a significant interaction between minute and outcome, suggesting that the devaluation effect increased across minutes of the test $\left(F_{(9,333)}=2.14 ; p<0.05\right)$. Thus, although the performance of the BLA rats was reduced relative to the other groups, their performance appeared to improve across the test and by the final minute of the session, evidence of a significant devaluation effect emerged $\left(F_{(1,19)}=13.35 ; p<0.01\right)$.

\section{Consumption test of specific satiety}

The data from the rewarded devaluation test suggest that, even when the outcomes were present during the test, the BLA rats did not show immediate sensitivity to the selective devaluation manipulation; their performance only became selective over the course of the session. It is possible, therefore, that this effect emerged because of new learning during the test. This may be because these animals have some trouble distinguishing between the two outcomes based on their sensory properties or because the devaluation treatment has a more general effect in these rats and acts to depress both responses. To further examine the ability of rats with BLA lesions to discriminate between the two outcomes and the effects of the selective devaluation procedure, independent of its effects on lever-press performance, we tested the rats' consumption of the two outcomes after devaluation by specific satiety.

The data for this test are presented in Figure 5. For the purpose of analysis and presentation, the amount of each type of food that the rats consumed (either grams of pellets or milliliters of sucrose) was converted to the equivalent number of outcomes (i.e., every $45 \mathrm{mg}$ of pellets is equivalent to one pellet outcome, whereas every $0.1 \mathrm{ml}$ of sucrose solution is equivalent to one sucrose outcome).

The results in Figure 5 suggest that, after prefeeding of one of 
the food outcomes, the rats ate more of a different food than the one that they had just eaten. Importantly, the magnitude of this effect was similar in all three groups, suggesting that the BLA rats could indeed distinguish between the two outcomes and showed a selective devaluation effect when measured in consumption. The statistical analysis supported this claim revealing no effect of group $\left(F_{(2,37)}=0.20 ; p>0.05\right.$; MSE $=1888.1)$, a significant effect of outcome (same vs different, $F_{(1,37)}=35.3 ; p<$ 0.01 ), and no interaction between group and outcome $\left(F_{(2,37)}=0.67 ; p>0.05\right)$.

\section{Pavlovian training}

To assess whether the animals learned the relationship between the stimuli and food deliveries, the number of magazine entries during the stimuli (CS) was compared with entries during a prestimulus interval of equal length. The training data are presented in the left panel of Figure 6, inspection of which suggests that pavlovian conditioning was similar in the three groups; all of the animals made more magazine entries during the CS presentations than during the prestimulus intervals. Preliminary analysis suggested that there was no effect of stimulus type (tone vs noise, $F$ value $<$ 1 ), and so the data are presented collapsed across stimulus type in $2 \mathrm{~d}$ blocks. ANOVA revealed no effect of group $\left(F_{(2,37)}=0.97\right.$; $p>0.05 ; \mathrm{MSE}=2897.0)$ but a main effect of training block, suggesting that more magazine entries were made as training progressed $\left(F_{(3,111)}=15.87 ; p<0.01\right)$, and of interval (CS vs pre-CS), which, as suggested by Figure 6, indicates that more entries were made during the CS than during the pre-CS interval $\left(F_{(1,37)}=568.81 ; p<0.01 ;\right.$ MSE $\left.=393.6\right)$. There was also an interaction between block and interval, suggesting that the difference in magazine entries between the CS and pre-CS intervals increases across blocks of training $\left(F_{(3,111)}=71.80 ; p<0.01\right)$. None of the other within-subject effects or interactions were significant $(F$ values $<1$; MSE $=393.6)$.

\section{Pavlovian-instrumental transfer test}

The objective of this test was to assess the impact of pavlovian cues for reward on instrumental performance and so for the purpose of analysis, the number of lever presses during the baseline or no-stimulus interval was subtracted from the number of lever presses during each of the stimuli. Positive numbers indicate, therefore, positive transfer (i.e., enhancement of responding above baseline), whereas numbers close to, or below, zero are indicative of reduced positive transfer. The data from the transfer tests are presented for each group in the right panels of Figure 6.

It is clear from the figure that the different lesions had very different effects on outcome-specific PIT. The sham rats show evidence of outcome-specific PIT increasing responding on the lever that, in training, delivered the same outcome as that predicted by the stimulus. Rats with $\mathrm{CN}$ lesions show a similar pattern of responding despite somewhat lower response rates. In contrast, rats with BLA lesions showed no evidence of any exci-

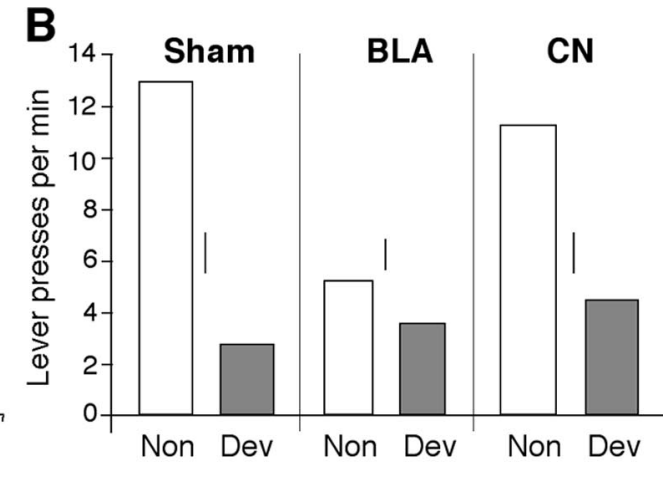

$\begin{array}{cc}5 & 6 \\ \text { Session } & 7\end{array}$

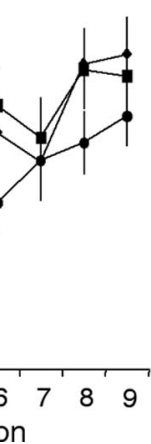

Figure 3. Experiment 1. $A$, Mean lever presses per minute ( $\pm 1 \mathrm{SEM})$ across sessions of instrumental training averaged across instrumental actions for each of the lesion groups. $B$, Mean lever presses per minute in the 10 min extinction test ( \pm 1 SE

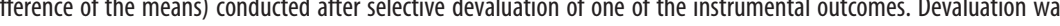
chieved using a specific-satiety procedure. Data are presented separately for the action that, in training, had delivered the now

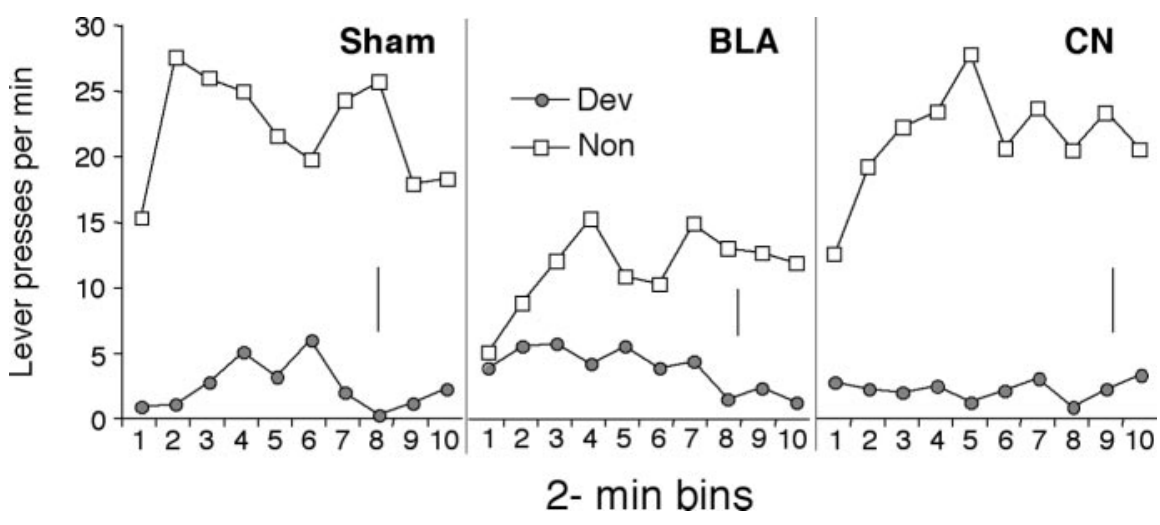

Figure 4. Experiment 1. Mean lever presses per minute in the 10 min rewarded devaluation test for each of the lesion groups ( $\pm 1 \mathrm{SE}$ of the difference of the means). Devaluation was achieved using a specific-satiety procedure as in the extinction test; however, performance of the two responses was rewarded with the outcomes that those responses had earned in training. Dev, Devalued; Non, nondevalued.

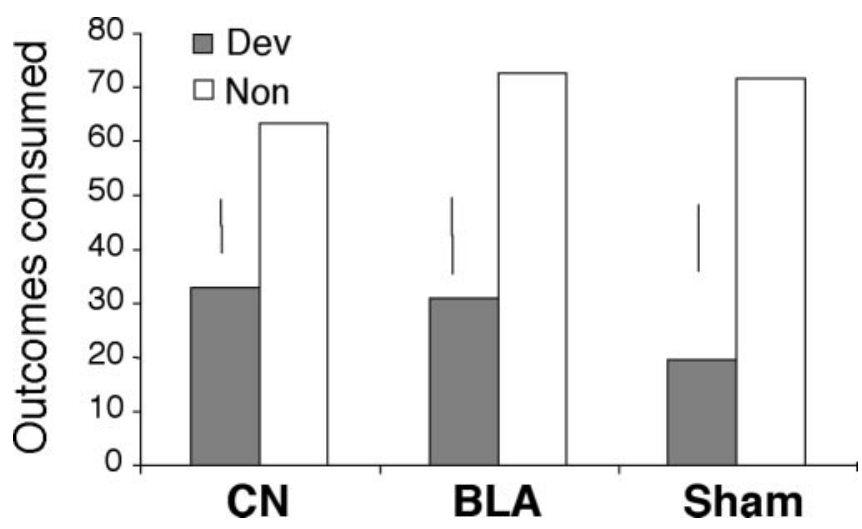

Figure 5. Experiment 1. Consumption test of specific-satiety induced devaluation ( $\pm 1 \mathrm{SE}$ of the difference of the means). Results are presented separately for consumption of the outcome that was the same as [devalued (Dev)] and different from [nondevalued (Non)] that outcome consumed immediately before the test phase and for each of the lesion groups.

tatory effect of the stimulus presentations. The statistical analysis confirmed this description; analysis of the test data revealed no main effect of group $\left(F_{(2,37)}=1.88 ; p>0.05\right.$; MSE $\left.=385.7\right)$, but there was a significant effect of stimulus (same vs different) $\left(F_{(1,37)}=38.14 ; p<0.01\right)$. Importantly, there was an interaction between stimulus and group $\left(F_{(2,37)}=4.46 ; p<0.05\right)$. As sug- 

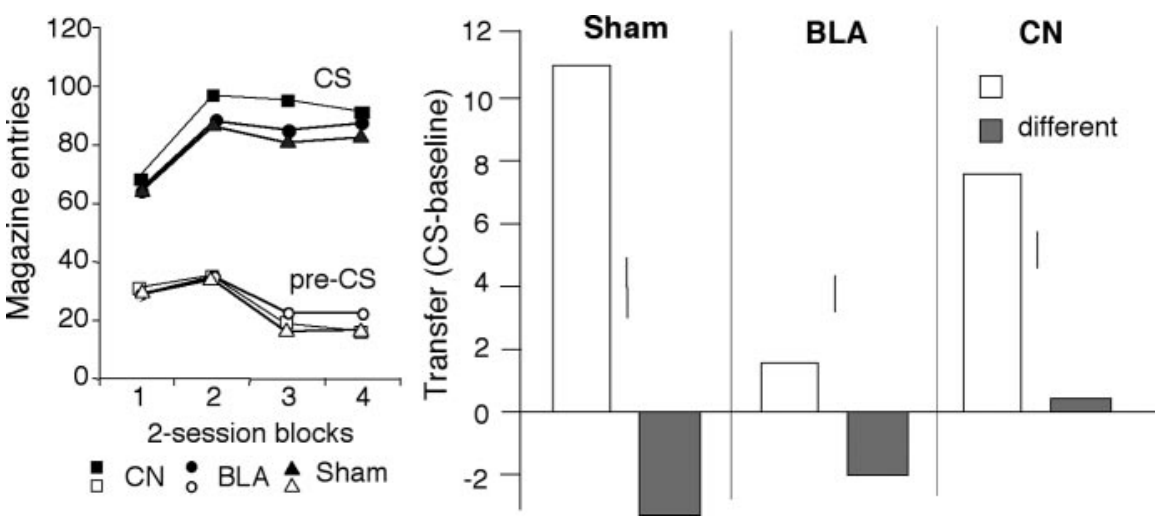

Figure 6. Experiment 1. Pavlovian conditioning and outcome-specific transfer. The left panel displays the mean number of magazine entries during the CS presentations and during the pre-CS intervals across days of pavlovian training for the three lesioned groups. The right panels display the mean number of lever presses per minute during the pavlovian-instrumental transfer test ( \pm 1 SE of the difference of the means). The number of responses during the baseline period was subtracted from the number of responses during the stimulus presentations to reveal the net excitatory effect of the stimuli. Same refers to the stimulus that predicts the same outcome as that delivered by the lever, whereas Different refers to the stimulus paired with the other outcome.

\begin{tabular}{c|c|c} 
training & conditioning & transfer test \\
\hline \multirow{2}{*}{$\mathrm{R} 1 \rightarrow \mathrm{O} 1 ; \mathrm{R} 2 \rightarrow \mathrm{O} 2$} & $\mathrm{~S} 1-\mathrm{O} 1$ & $\mathrm{~S} 1: \mathrm{R} 1, \mathrm{R} 2$ \\
& $\mathrm{~S} 2-\mathrm{O} 2$ & $\mathrm{~S} 2: \mathrm{R} 1, \mathrm{R} 2$ \\
& $\mathrm{~S} 3-\mathrm{O} 3$ & $\mathrm{~S} 3: \mathrm{R} 1, \mathrm{R} 2$ \\
\hline
\end{tabular}
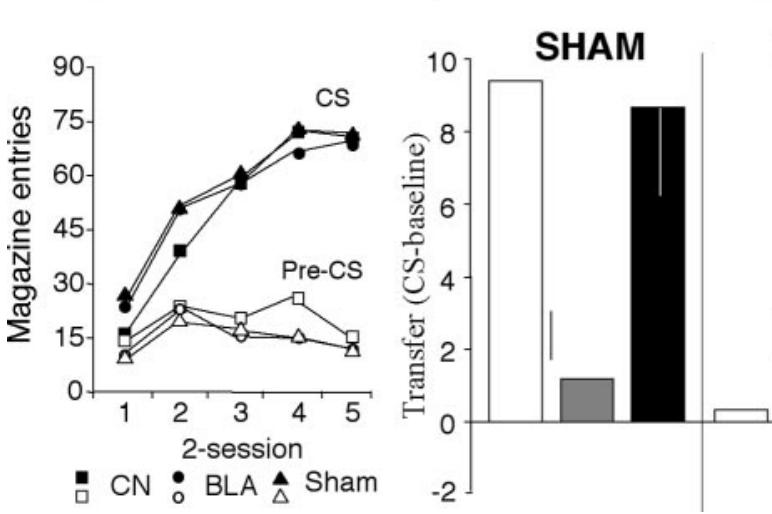

BLA

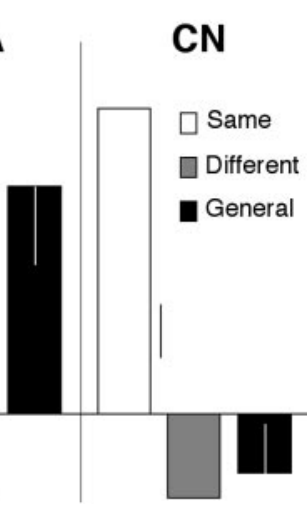

Figure 7. Experiment 2. General versus outcome-specific transfer. The design of experiment 2 is presented at the top: S1, S2, S3 refer to the clicker, tone, and white noise stimuli; R1 and R2 refer to the left and right lever press actions; and 01, 02, and 03 refer to the pellet, $20 \%$ sucrose, and $20 \%$ polycose outcomes. The identity of the stimuli, actions, and outcomes was counterbalanced across subjects in each group. The bottom left panel displays the mean number of magazine entries during the CS presentations and during the pre-CS intervals across days of pavlovian training for the three lesioned groups. The right panels display the mean number of lever presses per minute during the pavlovian-instrumental transfer test separately for the outcome-specific CSs (i.e., S1 and S2 \pm 1 SE of the difference of the means) and for the general excitatory stimulus (S3 \pm 1 SEM). The number of responses during the baseline period was subtracted from the number of responses during the stimulus presentations to reveal transfer (i.e., the net excitatory effect of the stimuli over baseline). Same refers to the stimulus that predicts the same outcome as that delivered by the lever, Different refers to the stimulus paired with the other outcome, and General refers to the stimulus paired with the outcome that was not earned during instrumental training.

gested by the figure, simple effects analyses revealed that whereas rats with $\operatorname{sham}\left(F_{(1,9)}=17.60 ; p<0.01\right)$ or $\mathrm{CN}\left(F_{(1,9)}=5.26 ; p<\right.$ $0.05)$ lesions showed evidence of outcome-specific PIT, rats with BLA lesions did not show an elevation in performance and did not respond differentially during presentation of the same and different stimuli $\left(F_{(1,19)}=0.10 ; p>0.05\right)$. Finally, these group differences arose during the stimuli and were not present during the baseline periods. The averaged responses per minute in the absence of the CS in experiment 1 were the following: group sham $=2.62$, group $\mathrm{BLA}=2.43$, and group $\mathrm{CN}=3.16$. These levels of responding did not differ significantly $(F$ values $<1$ ).
Experiment 2: effect of BLA and CN lesions on the general and specific forms of PIT

When examining the excitatory effects of reward-related stimuli on instrumental performance, there are two aspects that should be considered. First, a stimulus may produce a general enhancement in responding as a result of any generally arousing or motivational effects that the stimulus has acquired through its association with reward generally. Additionally, a stimulus may generate outcome-specific enhancement of a particular response as a result of a specific relationship with the unique sensory properties of a particular outcome. Data and resultant theories can be found to support both of these processes (Rescorla and Solomon, 1967; Trapold and Overmier, 1972; Overmier and Lawry, 1979; Colwill and Motzkin, 1994; Corbit and Balleine, 2003), and lesions of the amygdala could affect either or both of them.

However, understanding the difference between the generally arousing and the selective cuing properties of excitatory stimuli may be particularly important in understanding both the performance of the $\mathrm{CN}$ rats and in relating the current results to those of previous studies. As such, we implemented a procedure that allowed us to examine both the specific cuing and more generally arousing properties of stimuli concurrently. In the pavlovian training phase, two stimuli were paired with the two outcomes earned through instrumental performance. In addition, a third stimulus was paired with a third appetitive outcome that was distinct from the other outcomes with the aim that any excitatory effects of this third stimulus on instrumental performance would allow us to assess the contribution of any generally arousing impact of the pavlovian cues separately from the specific cuing function of the other stimuli. The design of this experiment is illustrated in the top panel of Figure 7.

\section{Instrumental training}

All groups acquired the lever-press responses, regardless of the outcomes used to reward this performance (outcome effect, $F$ values $<1$ ). Analysis revealed no effect of group $\left(F_{(2,29)}=0.94 ; p>0.05\right)$, a significant effect of day, suggesting that response rates increased across days of training $\left(F_{(6,174)}=62.4 ; p<0.01\right)$, and no interaction between day and group $\left(F_{(12,174)}=1.75 ; p>0.05\right)$.

\section{Pavlovian training}

The left panel of Figure 7 displays the data from the pavlovian training sessions. There was no effect of stimulus or of outcome type $(F$ values $<1)$, and so the data are presented collapsed across these factors in two-session blocks. As indicated by the figure, all 
groups acquired the magazine entry response; all of the rats in each of the groups made more magazine entries during the CS presentations than during the pre-CS intervals. ANOVA revealed no effect of group $\left(F_{(2,29)}=0.48 ; p>0.05 ; \mathrm{MSE}=1213.8\right)$ but a main effect of training block, suggesting that more magazine entries were made as training progressed $\left(F_{(4,116)}=78.14 ; p<\right.$ $0.01)$, and of interval (CS vs pre-CS) $\left(F_{(1,29)}=242.8 ; p<0.01\right)$, which, as suggested by Figure 7, confirms that more entries were made during the CS than during the pre-CS interval. There was also an interaction between block and interval, suggesting that the difference in magazine entries between the CS and pre-CS intervals increases across blocks of training $\left(F_{(4,116)}=92.14 ; p<\right.$ $0.01)$. None of the within-subjects effects or interactions involving the group factor were significant $(F$ values $<1$; MSE $=425.5)$.

\section{Pavlovian-instrumental transfer test}

The effect of the outcome-selective transfer (i.e., responding during S1 vs S2) and of general transfer (i.e., responding during S3) during the transfer test in experiment 2 is shown separately in the right panels of Figure 7. For the purposes of analyses, the number of responses in the absence of any stimuli (i.e., the intertrial interval) was subtracted from the responses during each of the stimuli to establish the net elevation above baseline produced by each of the pavlovian CSs. As the top panel of this figure shows, and as was found in experiment 1 , both the sham and the $\mathrm{CN}$ groups showed strong evidence of outcome-selective transfer. In contrast, no evidence of selective transfer was observed in the BLA group. In marked contrast to this pattern, however, responding during S3, the test of general transfer, found clear evidence of this form of transfer in both the sham and the BLA groups, whereas no clear general transfer was observed in the $\mathrm{CN}$ group.

The overall ANOVA found no effect of group $\left(F_{(2,29)}=0.57\right.$; $p>0.05 ; \mathrm{MSE}=385.7)$ but revealed a significant effect of stimulus (same, different, and general; $F_{(2,58)}=4.16 ; p<0.05$ ) and, importantly, a significant interaction between group and stimulus $\left(F_{(4,58)}=2.68 ; p<0.05\right)$. Simple effects analysis revealed that whereas responding during the same versus different stimuli differed in group sham $\left(t_{(11)}=2.58 ; p<0.05\right)$ and group CN $\left(t_{(9)}=\right.$ $2.47 ; p<0.05)$, there was no reliable effect in group BLA $\left(t_{(9)}=\right.$ $-0.38 ; p>0.05)$. In contrast, using Fishers PLSD post hoc tests conducted on mean responding during stimulus S3, whereas responding did not differ between the sham and the BLA groups $(p>0.05)$, responding differed significantly between the sham and the CN groups $(p<0.38)$. The group effects were not a product of any differences in responding during the baseline periods. The averaged responses per minute in the absence of the CS in experiment 2 were the following: group sham $=2.51$, group $\mathrm{BLA}=3.06$, and group $\mathrm{CN}=3.55$. These levels of responding did not differ significantly $(F$ value $<1)$. Experiment 2 revealed, therefore, evidence of a double dissociation between BLA and CN lesions with respect to their effects on the different forms of PIT. Whereas lesions of the $\mathrm{CN}$ spared outcome-selective transfer but abolished general transfer, lesions of the BLA abolished outcomeselective transfer but had no effect on general transfer.

\section{Discussion}

The results of these experiments establish that the BLA and CN play distinct roles in the way reward-related processes control instrumental performance. Specifically, these data suggest that, in instrumental conditioning, the BLA, rather than the $\mathrm{CN}$, is the more critical substrate for controlling the selective cuing effects related to the identity of a particular outcome, whereas the $\mathrm{CN}$ appears to be more generally involved in appetitive arousal. Experiment 1 demonstrated that the selective effects of outcome devaluation and of pavlovian CSs on instrumental performance were abolished by lesions of the BLA but were spared by lesions of the $\mathrm{CN}$. In experiment 2, we developed a procedure using which we were able to generate both outcome-specific and general PIT in the same animal using two CSs (S1 and S2) to predict the outcomes earned by the actions in training and a third CS (S3) to predict an outcome that was not earned by either of the actions. In sham rats, $\mathrm{S} 1$ and $\mathrm{S} 2$ produced outcome-selective PIT, electively elevating one or other instrumental response, whereas S3 was found to generally increase the performance of both actions above baseline. Importantly, whereas lesions of the BLA abolished outcome-specific PIT, they did not affect the general increase in performance produced by S3. In contrast, lesions of the $\mathrm{CN}$ spared outcome-specific PIT but abolished the general excitatory effect of S3 on instrumental performance. Hence, this study provides evidence that the specific and the general forms of PIT can be doubly dissociated at the level of the amygdala. Hence, experiment 2 established that the source of the difference between the reported effects of BLA and CN lesions on PIT (Blundell et al., 2001; Hall et al., 2001; Holland and Gallagher, 2003) lies in the form of transfer induced by the distinct instrumental training protocols used in the different series of experiments.

The influence of pavlovian cues on instrumental performance The double dissociation observed in experiment 2 suggests that what were previously only descriptively distinct forms of transfer (cf. Dickinson and Balleine, 2002) are in fact mediated by independent processes and point to the fact that pavlovian cues can influence instrumental performance through two routes. One of these involves the ability of stimuli associated with rewarding outcomes to influence performance indirectly by increasing motivational arousal. The other involves the ability of CSs to activate the memory of the sensory-specific features of the outcome or reward used in instrumental conditioning. But, as the current evidence makes clear, it cannot be that these features serve merely to gate what is otherwise general motivational arousal as has sometimes been claimed (Dickinson and Dearing, 1979). Rather, it appears that retrieval of the sensory features of an outcome influences instrumental performance by retrieving the action associated with that outcome, something that experiment 2 suggests results in the inhibition of other actions. The presentation of a stimulus paired with an outcome that was different from that earned by a response failed to enhance performance, although it was clearly an appetitive CS. At present, this inhibitory influence on action selection is not well understood although, as Colwill and Rescorla (1990), Rescorla (1994), and, recently, Holland (2004) have all shown, outcome devaluation does not affect selective transfer, suggesting that selective PIT may depend on the information conveyed by a stimulus rather than on its motivational effects.

\section{The role of the amygdala in instrumental conditioning}

The current series of experiments found that lesions of the BLA eliminate the selective excitatory effect of reward-related stimuli on instrumental responding but left the acquisition of pavlovian and instrumental conditioning otherwise intact. This finding is consistent with other data. For example, BLA lesions have been shown to impair second-order conditioning, and this impairment has often been interpreted as evidence that the BLA is required in order for stimuli to acquire incentive value by virtue of their associations with the motivational properties of primary 
reward (Hatfield et al., 1996; Setlow et al., 2002). Similarly, BLA lesions have been shown to produce impairments in conditioned reinforcement, a situation in which the motivational value of a $\mathrm{CS}$, acquired through pairings with a primary reward, is able to reinforce and maintain instrumental performance (Cador et al., 1989; Burns et al., 1993; Whitelaw et al., 1996).

Also consistent with previous reports (Hatfield et al., 1996; Balleine et al., 2003), the current study found that lesions of the BLA, but not the CN, reduced the rats' sensitivity to the selective devaluation of an instrumental outcome, although Balleine et al. (2003) found that their BLA lesioned rats were generally unaffected by devaluation, whereas, in experiment 1 , responding appeared to be more generally reduced. The fact that the rats were tested in a choice situation, however, makes it difficult to compare individual actions between groups; for example, the overall operant rate of the BLA, but not sham, group in the current experiment could have been reduced by interference produced by the changeover between actions. Nevertheless, as observed by Balleine et al. (2003), the data from the rewarded test (compare Fig. 5) suggest that performance can be rescued somewhat if the outcomes are delivered during the test and, likewise, that BLA rats were similar to shams when consumption was used as a measure of sensitivity to the devaluation treatment. The effect of BLA lesions on devaluation cannot, therefore, be attributable simply to insensitivity to the devaluation manipulation or an inability to discriminate between the instrumental actions or outcomes. Rather, it is possible that, although able to form associations between their responses and specific outcomes, BLA rats do not form associations between their responses and the specific affective or incentive properties of those outcomes.

Alternatively, it has been suggested that BLA lesions may force animals to acquire responses based on stimulus-response (S-R) rather than on CS- unconditioned stimulus or response-outcome associations (Setlow et al., 2002; Balleine et al., 2003). Responses governed by S-R associations are generally thought to be impervious to devaluation manipulations, because the outcome itself is not represented within the associative structure controlling responding, and so changes in its value have no immediate effect on performance (Adams, 1982; Dickinson and Balleine, 1994). To the extent that S-R associations are guiding performance, specific satiety-induced devaluation should be predicted to produce a general decrease in response rates caused by the reduction in hunger, and hence drive, resulting from the prefeeding treatment (Dickinson et al., 1995), which is exactly what was observed in the devaluation test in the BLA group. The S-R account can also help to explain why the performance of the BLA group improved when the outcomes were delivered during the rewarded test. For example, the performance in the rewarded test could reflect new learning; the strength of existing $\mathrm{S}-\mathrm{R}$ associations being alternatively strengthened by the nondevalued outcome and weakened by the devalued outcome (Balleine et al., 2003).

The characterization of the function of the $\mathrm{CN}$ is made difficult because, generally, rats with these lesions seemed able to perform reasonably well in most of the tasks examined here. That is not to say, however, that their performance was entirely normal. Although rats in the $\mathrm{CN}$ group showed evidence of selective responding in both of the PIT tests, the magnitude of the effects observed was always numerically attenuated relative to sham rats. And, of course, the $\mathrm{CN}$ rats failed to show evidence of a general transfer effect. The $\mathrm{CN}$ has strong projections to the hypothalamus, reticular formation, and brainstem nuclei, and so it is often argued to be involved in the arousal of behavioral, autonomic, and endocrine responses (Cardinal et al., 2002). This is consistent with other reports that $\mathrm{CN}$ lesions produce deficits in attentional tasks (Holland et al., 2000, 2001; McDannald et al., 2004), CS processing or associability (Holland and Gallagher, 1993a,b), and tasks that have assessed arousal (Merali et al., 1998; Wilhelmi et al., 2001).

The CN has also been argued to exert control over the midbrain dopaminergic afferents of the nucleus accumbens (Hall et al., 2001) and, in this way, to modulate the reward and performance-related functions of the ventral striatum. Indeed, Hall et al. (2001) reported that lesions of the accumbens core, but not the accumbens shell, had quite similar effects to those of the $\mathrm{CN}$ on nonselective, general PIT. It contrast, in a previous report (Corbit et al., 2001), we found, in apparently direct opposition to Hall et al. (2001), that outcome-specific transfer is abolished by lesions of shell but spared by lesions of core. Given the results of the current study, however, it would seem likely that resolution to this conflict also lies in the distinct forms of transfer generated in the two studies. Generally, therefore, this dissociation between the general excitatory effects and the more specific cuing functions of reward-related stimuli in instrumental conditioning may help reconcile not only the inconsistencies in previous reports of amygdala lesions but also those involving lesions of the nucleus accumbens and specifically the distinct functions associated with the core and shell subregions of that structure.

\section{References}

Adams CD (1982) Variations in the sensitivity of instrumental responding to reinforcer devaluation. Q J Exp Psychol B 34:77-98.

Balleine BW (1994) Asymmetrical interactions between thirst and hunger in Pavlovian-instrumental transfer. Q J Exp Psychol B 47:211-231.

Balleine BW (2001) Incentive processes in instrumental conditioning. In: Handbook of contemporary learning theories (Mowrer R, Klein S, eds), pp 307-366. Hillsdale, NJ: Lawrence Erlbaum Associates.

Balleine BW (2004) Incentive behavior. In: The behavior of the laboratory rat: a handbook with tests, Chap 41 (Whishaw IQ, Kolb B, eds) pp 436446. Oxford: Oxford UP.

Balleine BW, Killcross S, Dickinson A (2003) The effect of lesions of the basolateral amygdala on instrumental conditioning. J Neurosci 23:666-675.

Blundell P, Hall G, Killcross S (2001) Lesions of the basolateral amygdala disrupt selective aspects of reinforcer representation in rats. J Neurosci 21:9018-9026.

Burns LH, Robbins TW, Everitt BJ (1993) Differential effects of excitotoxic lesions of the basolateral amygdala, ventral subiculum and medial prefrontal cortex on responding with conditioned reinforcement and locomotor activation potentiated by intra-accumbens infusions of D-amphetamine. Behav Brain Res 55:167-183.

Cador M, Robbins TW, Everitt BJ (1989) Involvement of the amygdala in stimulus-reward associations: interactions with the ventral striatum. Neuroscience 30:77-88.

Cardinal RN, Parkinson JA, Hall J, Everitt BJ (2002) Emotion and motivation: the role of the amygdala, ventral striatum, and prefrontal cortex. Neurosci Biobehav Rev 26:321-352.

Colwill RM, Motzkin DK (1994) Encoding of the unconditioned stimulus in Pavlovian conditioning. Anim Learn Behav 22:384-394.

Colwill RM, Rescorla RA (1990) Effect of reinforcer devaluation on discriminative control of instrumental behavior. J Exp Psychol Anim Behav Process 16:40-47.

Corbit LH, Balleine BW (2003) Pavlovian and instrumental incentive processes have dissociable effects on components of a heterogeneous instrumental chain. J Exp Psychol Anim Behav Process 29:99-106.

Corbit L, Muir J, Balleine BW (2001) The role of the nucleus accumbens in instrumental conditioning: evidence for a functional dissociation between accumbens core and shell. J Neurosci 21:3251-3260.

Dayan P, Balleine BW (2002) Reward, motivation and reinforcement learning. Neuron 36:285-298.

Dickinson A, Balleine BW (1994) Motivational control of goal-directed action. Anim Learn Behav 22:1-18. 
Dickinson A, Balleine BW (1995) Motivational control of instrumental action. Curr Dir Psychol Sci 4:162-167.

Dickinson A, Balleine BW (2002) The role of learning in motivation. In: Steven's handbook of experimental psychology, Vol 3, Learning, motivation and emotion, Ed 3 (Gallistel CR, ed), pp 497-533. New York: Wiley.

Dickinson A, Dawson GR (1987) Pavlovian processes in the motivational control of instrumental performance. Q J Exp Psychol B 39:201-213.

Dickinson A, Dearing MF (1979) Appetitive-aversive interactions and inhibitory processes. In: Mechanism of learning and motivation (Dickinson A, Boakes RA, eds), pp 203-231. Hillsdale, NJ: Lawrence Erlbaum Associates.

Dickinson A, Balleine BW, Watt A, Gonzales F, Boakes RA (1995) Motivational control after extended instrumental training. Anim Learn Behav 23:197-206.

Hall J, Parkinson JA, Connor TM, Dickinson A, Everitt BJ (2001) Involvement of the central nucleus of the amygdala and nucleus accumbens core in mediating Pavlovian influences on instrumental behaviour. Eur J Neurosci 13:1984-1992.

Hatfield T, Han J, Conley M, Gallagher M, Holland P (1996) Neurotoxic lesions of basolateral, but not central, amygdala interfere with Pavlovian second-order conditioning and reinforcer devaluation effects. J Neurosci $16: 5256-5265$.

Holland PC (2004) Relations between Pavlovian-instrumental transfer and reinforcer devaluation. J Exp Psychol Anim Behav Process 30:104-117.

Holland PC, Gallagher M (1993a) Effects of amygdala central nucleus lesions on blocking and unblocking. Behav Neurosci 107:235-245.

Holland PC, Gallagher M (1993b) Amygdala central nucleus lesions disrupt increments, but not decrements, in conditioned stimulus processing. Behav Neurosci 107:246-253.

Holland PC, Gallagher M (2003) Double dissociation of the effects of lesions of the basolateral and central amygdala on conditioned stimuluspotentiated feeding and Pavlovian-instrumental transfer. Eur J Neurosci $17: 1680-1694$.

Holland PC, Han JS, Gallagher M (2000) Lesions of the amygdala central nucleus alter performance on a selective attention task. J Neurosci 20:6701-6706.
Holland PC, Chik Y, Zhang Q (2001) Inhibitory learning tests of conditioned stimulus associability in rats with lesions of the amygdala central nucleus. Behav Neurosci 115:1154-1158.

McDannald M, Kerfoot E, Gallagher M, Holland PC (2004) Amygdala central nucleus function is necessary for learning but not expression of conditioned visual orienting. Eur J Neurosci 20:240-248.

Merali Z, McIntosh J, Kent P, Michaud D, Anisman H (1998) Aversive and appetitive events evoke the release of corticotropin-releasing hormone and bombesin-like peptides at the central nucleus of the amygdala. J Neurosci 18:4758-4766.

Overmier JB, Lawry JA (1979) Pavlovian conditioning and the mediation of behavior. In: The psychology of learning and motivation, Vol 13 (Bower GH, ed), pp 1-55. New York: Academic.

Paxinos G, Watson C (1998) The rat brain in stereotaxic coordinates. San Diego: Academic.

Rescorla RA (1994) Transfer of instrumental control mediated by a devalued outcome. Anim Learn Behav 22:27-33.

Rescorla RA, Solomon RL (1967) Two-process learning theory: relationship between Pavlovian conditioning and instrumental learning. Psychol Rev 74:151-182

Setlow B, Gallagher M, Holland PC (2002) The basolateral complex of the amygdala is necessary for acquisition but not expression of CS motivational value in appetitive Pavlovian second-order conditioning. Eur J Neurosci 15:1841-1853.

Trapold MA, Overmier JB (1972) The second learning process in instrumental learning. In: Classical conditioning II: current research and theory (Black AA, Prokasy WF, eds), pp 427-452. New York: Appleton-Century-Crofts.

Whitelaw RB, Markou A, Robbins TW, Everitt BJ (1996) Excitotoxic lesions of the basolateral amygdala impair the acquisition of cocaine-seeking behavior under a second-order schedule of reinforcement. Psychopharmacology 127:213-224.

Wilhelmi E, Linke R, de Lima AD, Pape HC (2001) Axonal connections of thalamic posterior paralaminar nuclei with amygdaloid projection neurons to the cholinergic basal forebrain in the rat. Neurosci Lett 315:121-124. 